\title{
The ATLAS Electron and Photon Trigger Performance in Run-2
}

\author{
Daniela Köck \\ on behalf of the ATLAS collaboration
}

8th International Conference on New Frontiers in Physics (ICNFP 2019)
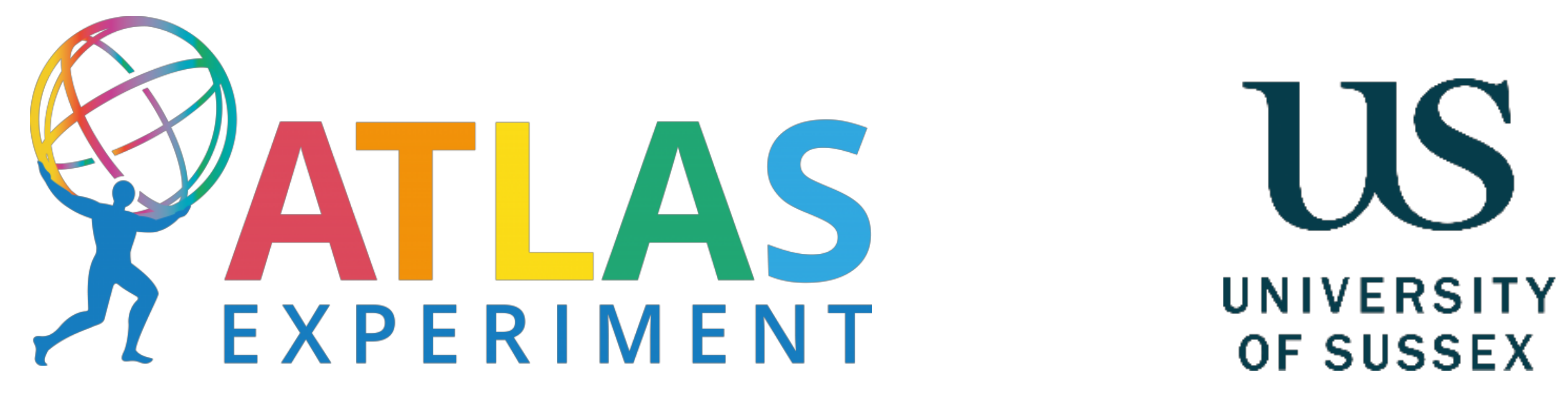


\section{Overview}

\section{Introduction}

The ATLAS trigger system in a nutshell, Ringer algorithm offline electron and photon reconstruction

electron and photon trigger efficiency measurements

$Z$ tag-and-probe method, $Z$ radiative decay method, Bootstrap method

Performance of electron and photon triggers during Run-2

Level-1 performance, electron and photon trigger evolution,

trigger in heavy ion collisions

Conclusions and Outlook 


\section{The ATLAS trigger system in a nutshell}

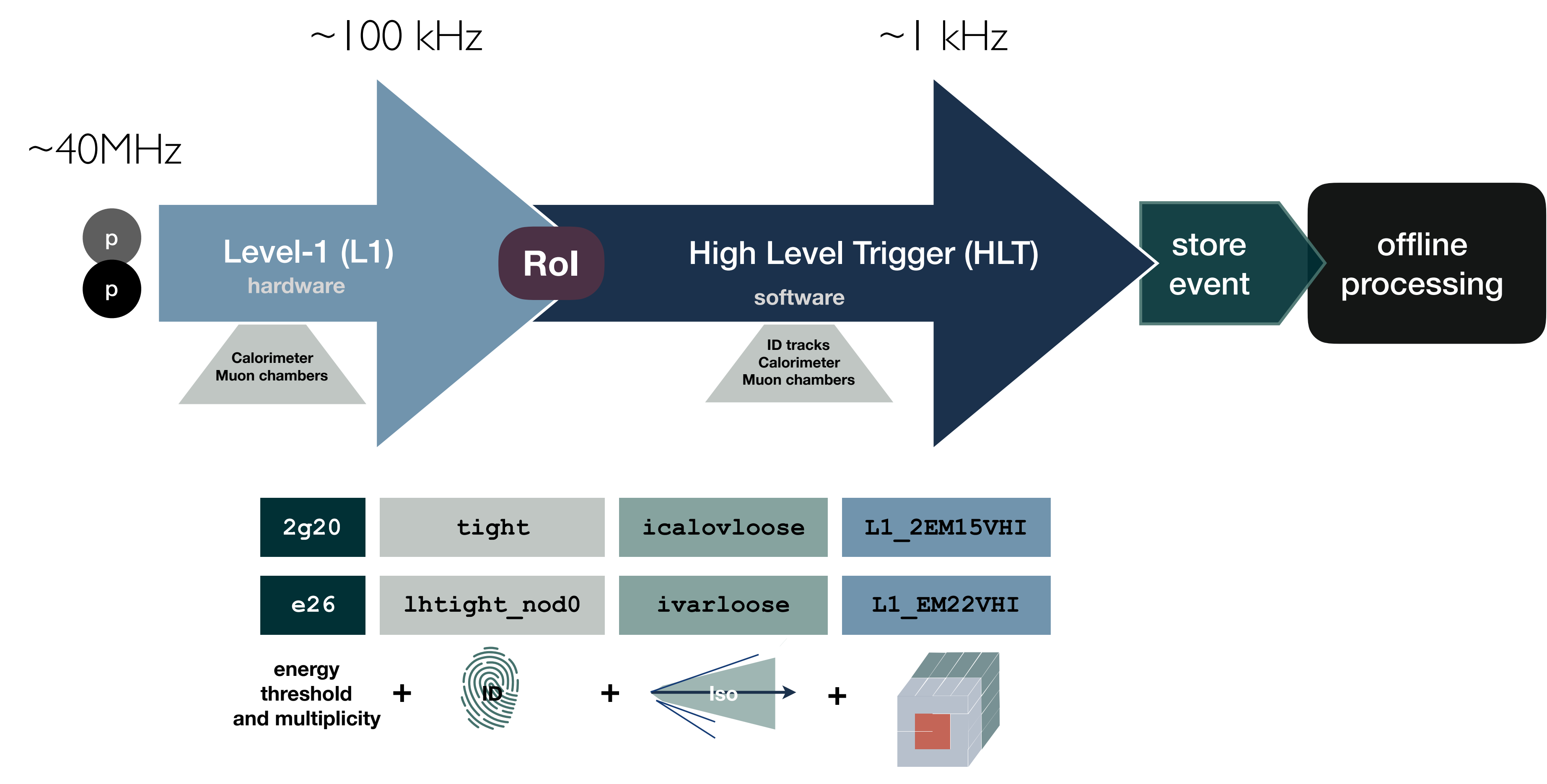

\section{Level-1}

$2 \times 2$ trigger tower cluster as Rol in EM calorimeter

$\mathbf{V}$ : varying ET threshold within -2 and $+3 \mathrm{GeV}$ of nominal threshold

$\mathbf{H}$ : veto on hadronic leakage

I: ET dependent isolation of cluster in EM calorimeter

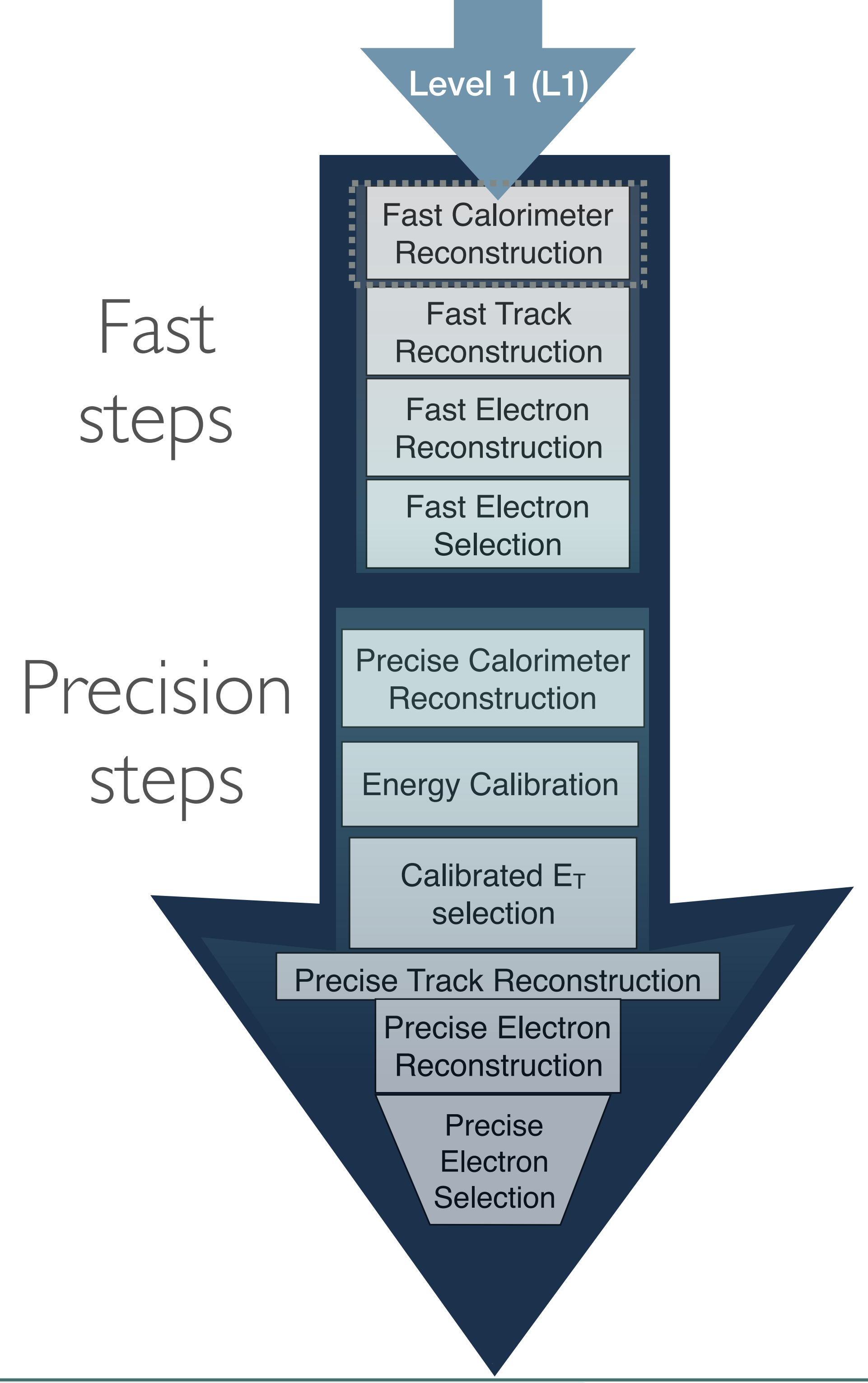




\section{Ringer algorithm}

- used from 2017 on to trigger electrons

(Fast Calorimeter step) with $\mathrm{E}_{\mathrm{T}}>15 \mathrm{GeV}$

- use lateral shower development

- concentric ring energy sums in each calorimeter layer

- transverse energy in each ring normalised to total transverse energy in the Rol

- ring energies fed into multilayer perceptron (MLP) neutral networks

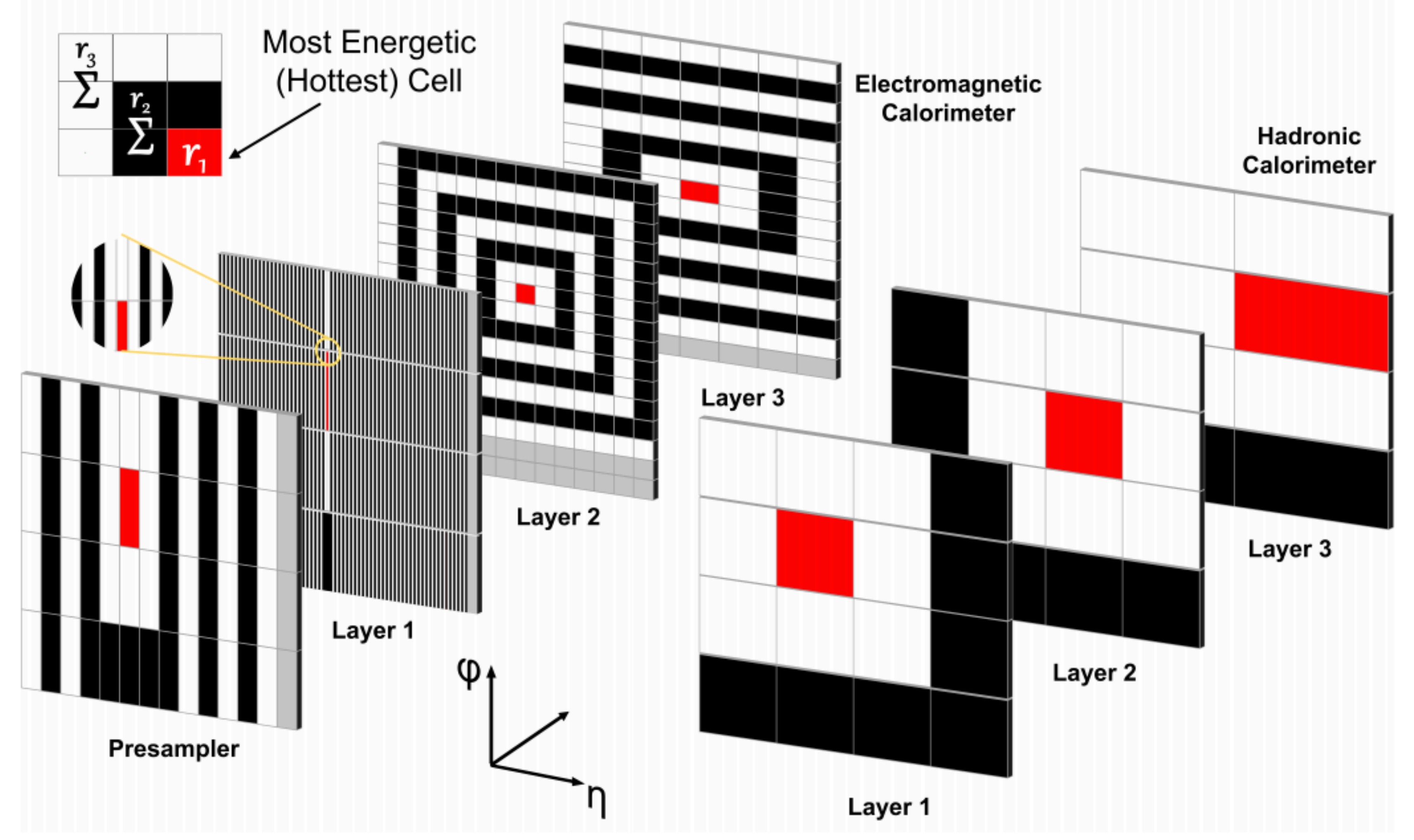

Ringer increases Fast Calorimeter step reconstruction time, but reduces input candidates to the tracking $\rightarrow$ significantly reduced CPU demand (50\% CPU reduction for the lowest $\mathrm{P}_{\mathrm{T}}$ unprescaled single electron trigger) 


\section{'Offline' electron and photon reconstruction and identification}

\section{Electrons}

- Identification based on a likelihood discriminator

- 'loose', 'medium', 'tight' working points considered

- using GSF ( Gaussian-Sum Filter) as a generalisation of the Kalman fitter,

better account for energy loss in Inner Detector

\section{Photons}

- identification based on calorimetric variables

- two identification working points, 'loose' and 'tight'

- 'loose' relying on shower shapes in the second electromagnetic calorimeter layer and hadronic deposits

- 'tight' including first layer of electromagnetic calorimeter

using Supercluster to improve electron and photon energy reconstruction in cases with Bremsstrahlung or pair production

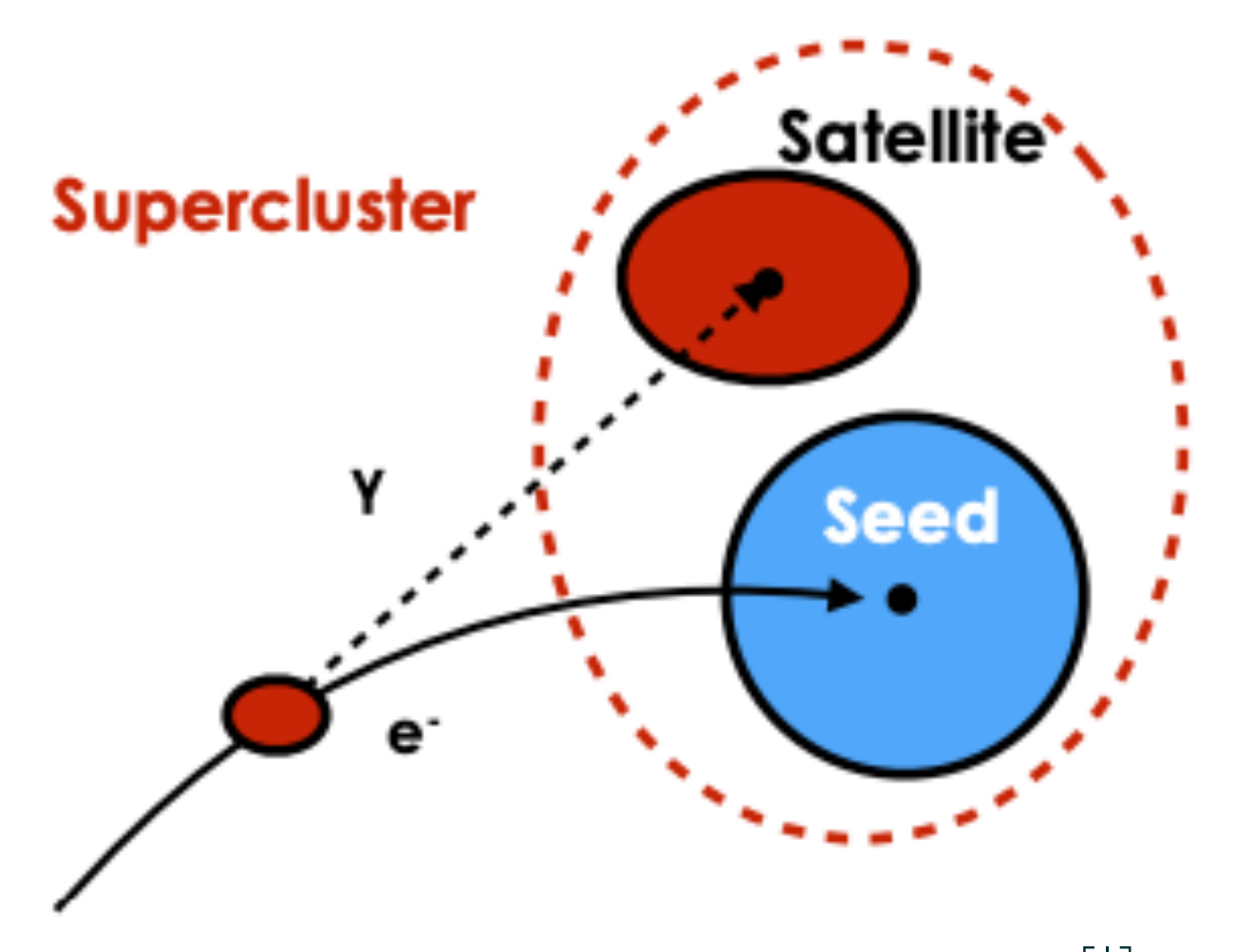




\section{Performance measurement techniques - electrons}

\section{$Z$ tag-and-probe method}

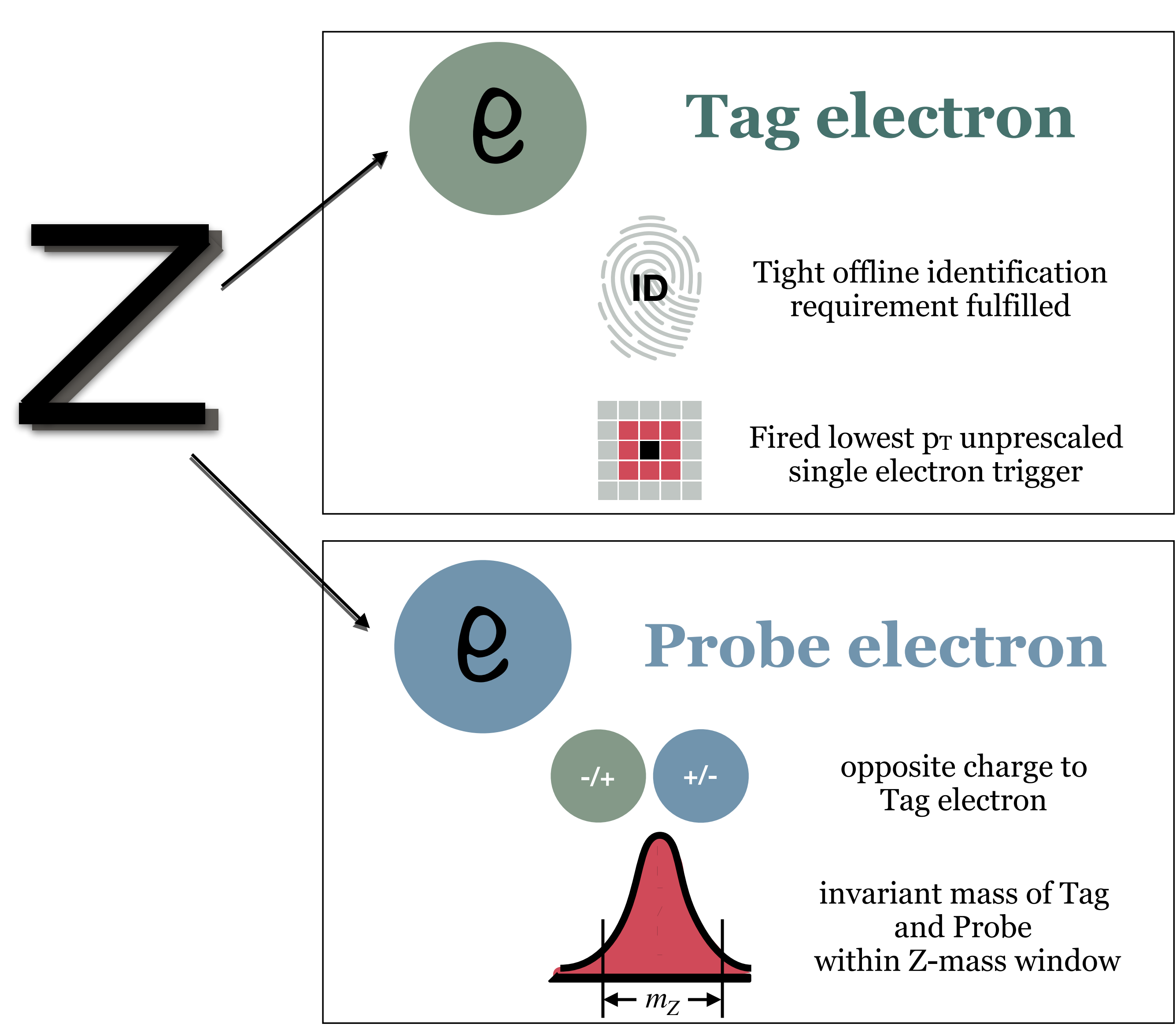

$\epsilon_{\text {total }}=\epsilon_{\text {offline }} \times \epsilon_{\text {trig }}=\left(\frac{N_{\text {offline }}}{N_{\text {all }}}\right) \times\left(\frac{N_{\text {trig }}}{N_{\text {offline }}}\right)$

compute trigger efficiency

with respect to offline electron definitions

\section{systematic uncertainties}

- given by varying tag definition, Z-mass window and background subtraction method

- central value is average of variations 


\section{Performance measurement techniques - photons}

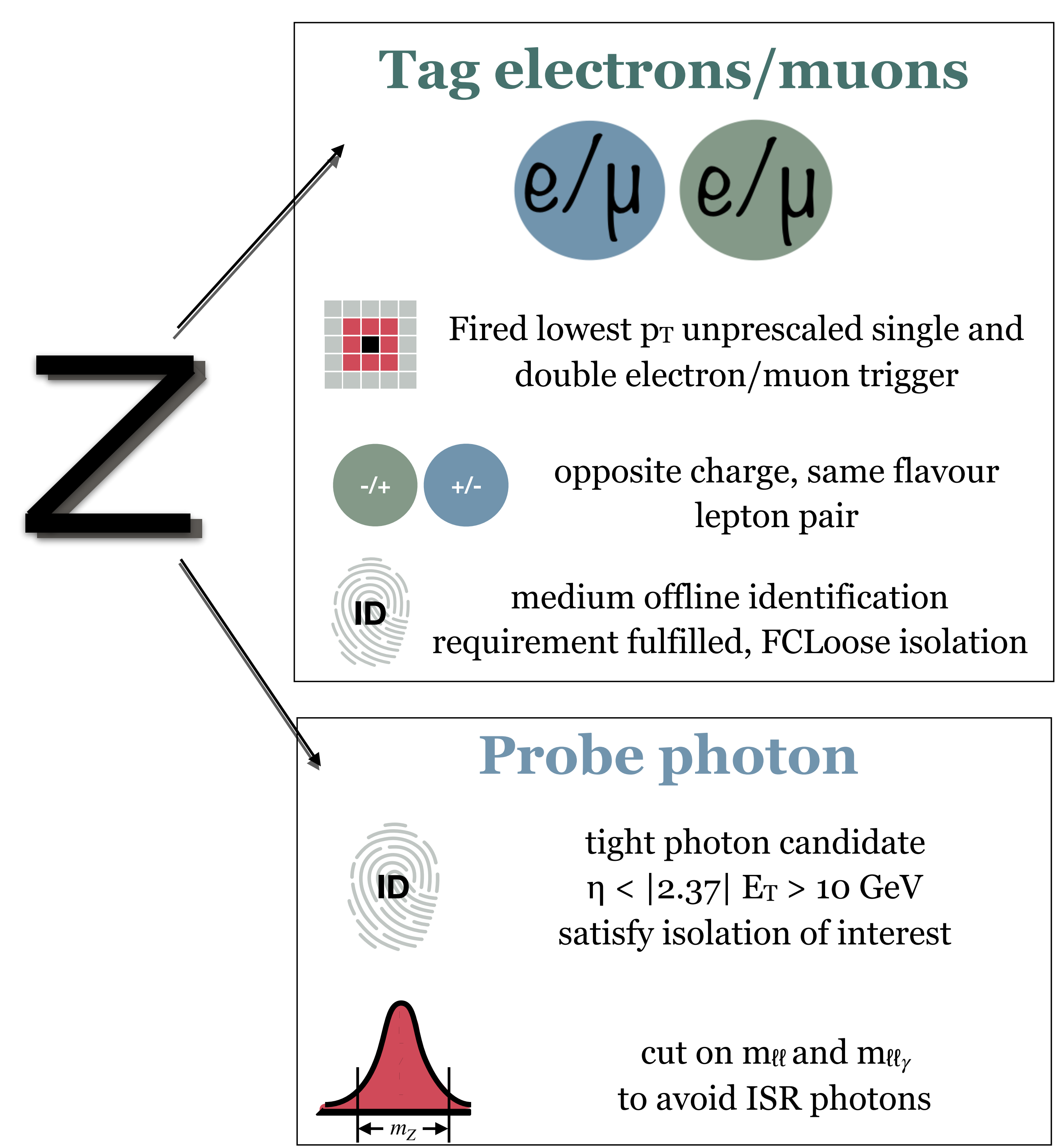

\section{$\mathrm{Z}$ radiative decay method}

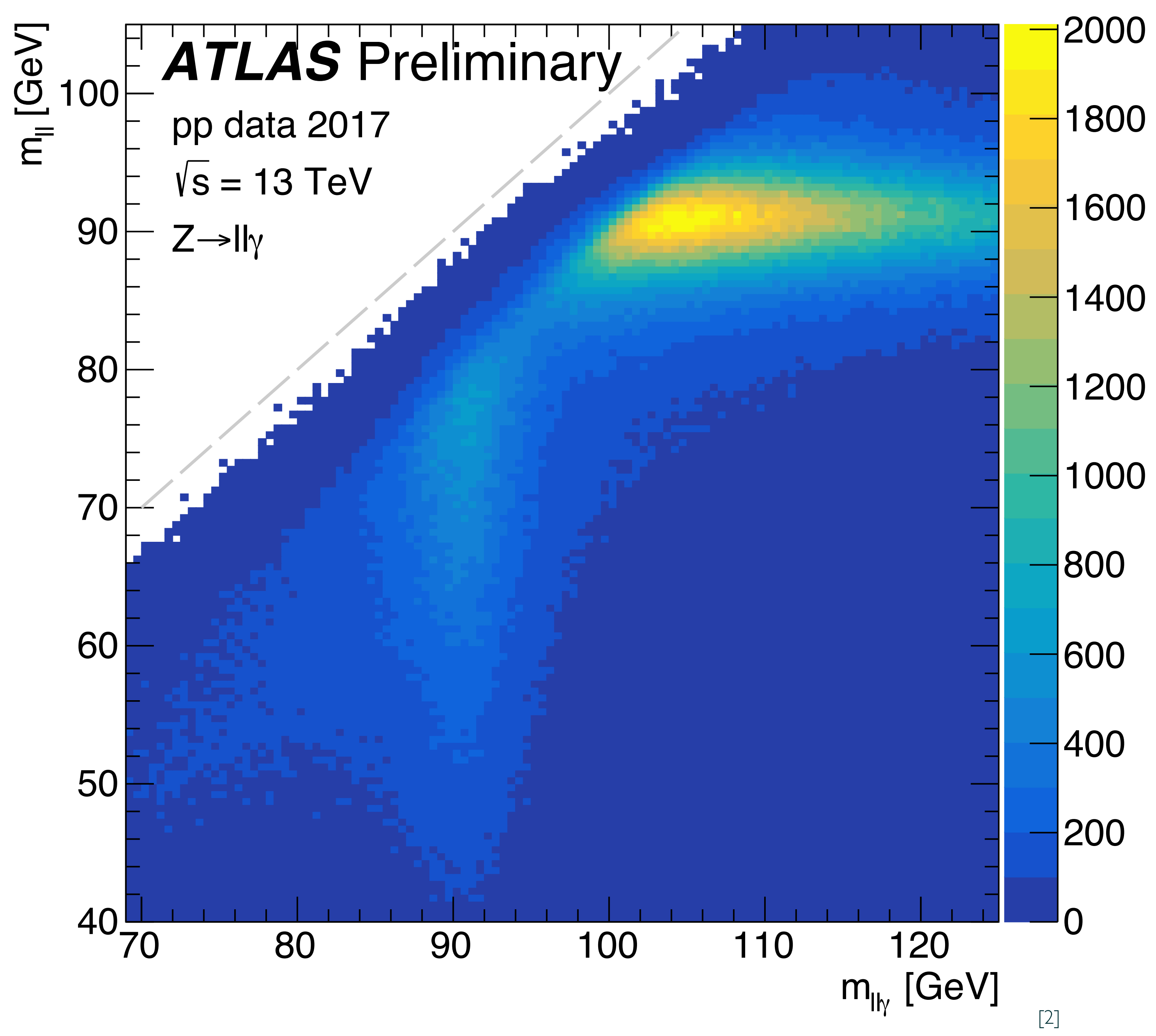




\section{Performance measurement techniques - photons}

\section{Bootstrap method}

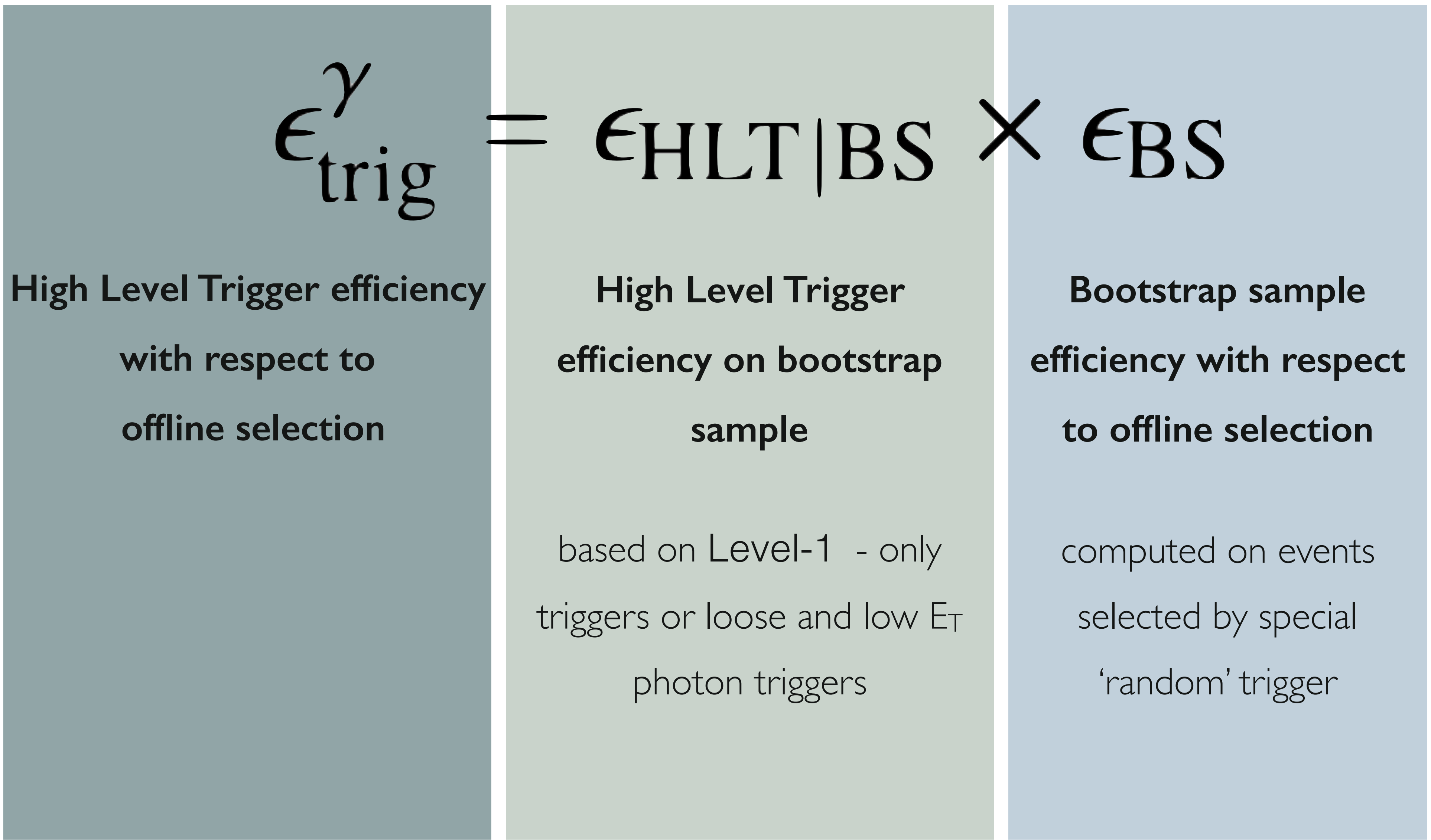

- large, uncontrolled background contamination (main source of uncertainty)

- systematic uncertainty as difference between data and simulated $H \rightarrow \gamma \gamma$ events 


\section{Level-1 trigger performance}

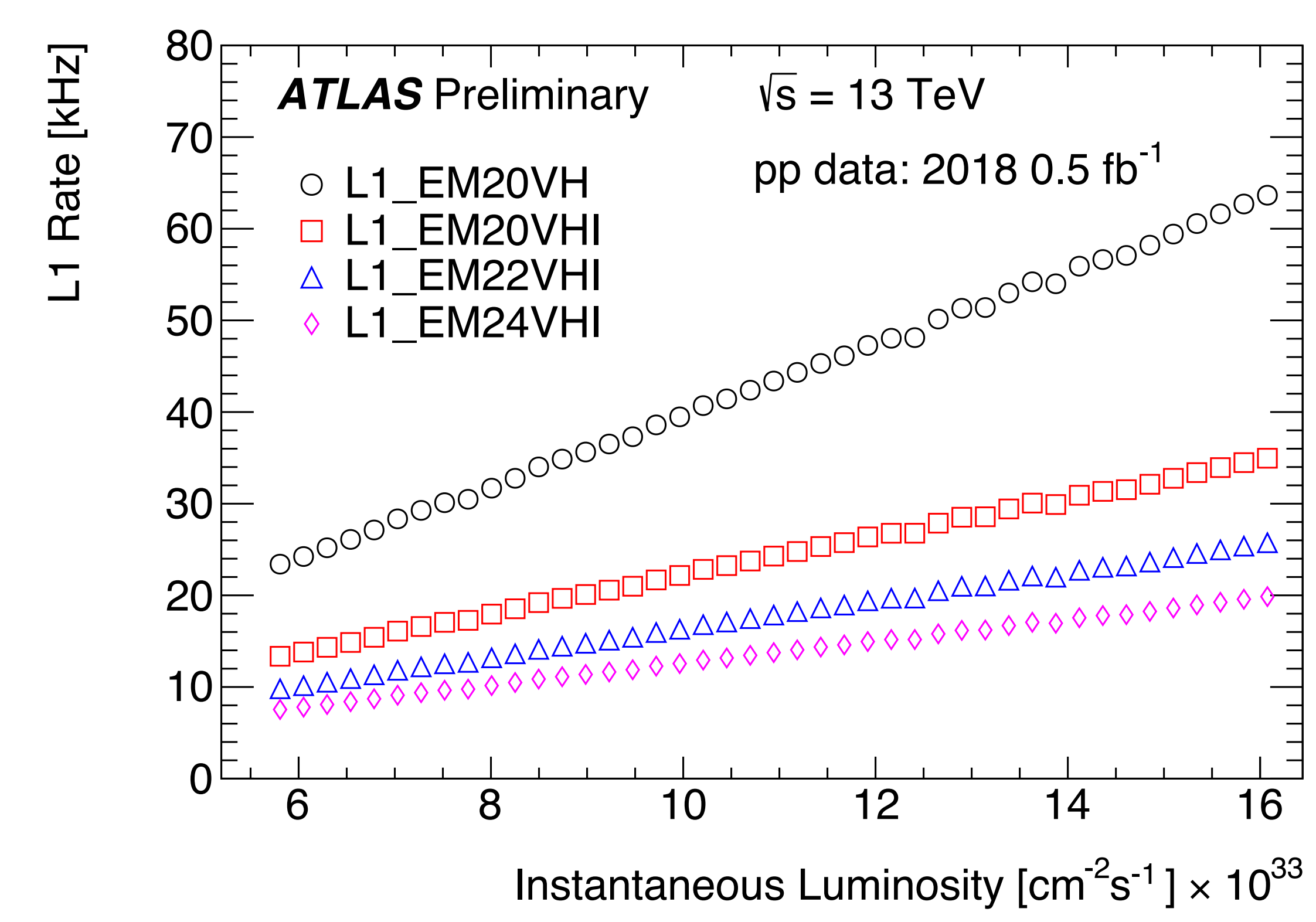

[2]
- linear increase of rate with instantaneous luminosity

- single Level-1 EM triggers: raise of threshold by $2 \mathrm{GeV}$ leads to $25 \%$ decrease in rate

rate reduction of $\sim 44 \%$ per leg by including electromagnetic calorimeter isolation at Level-1, efficiency decrease up to $5 \%$
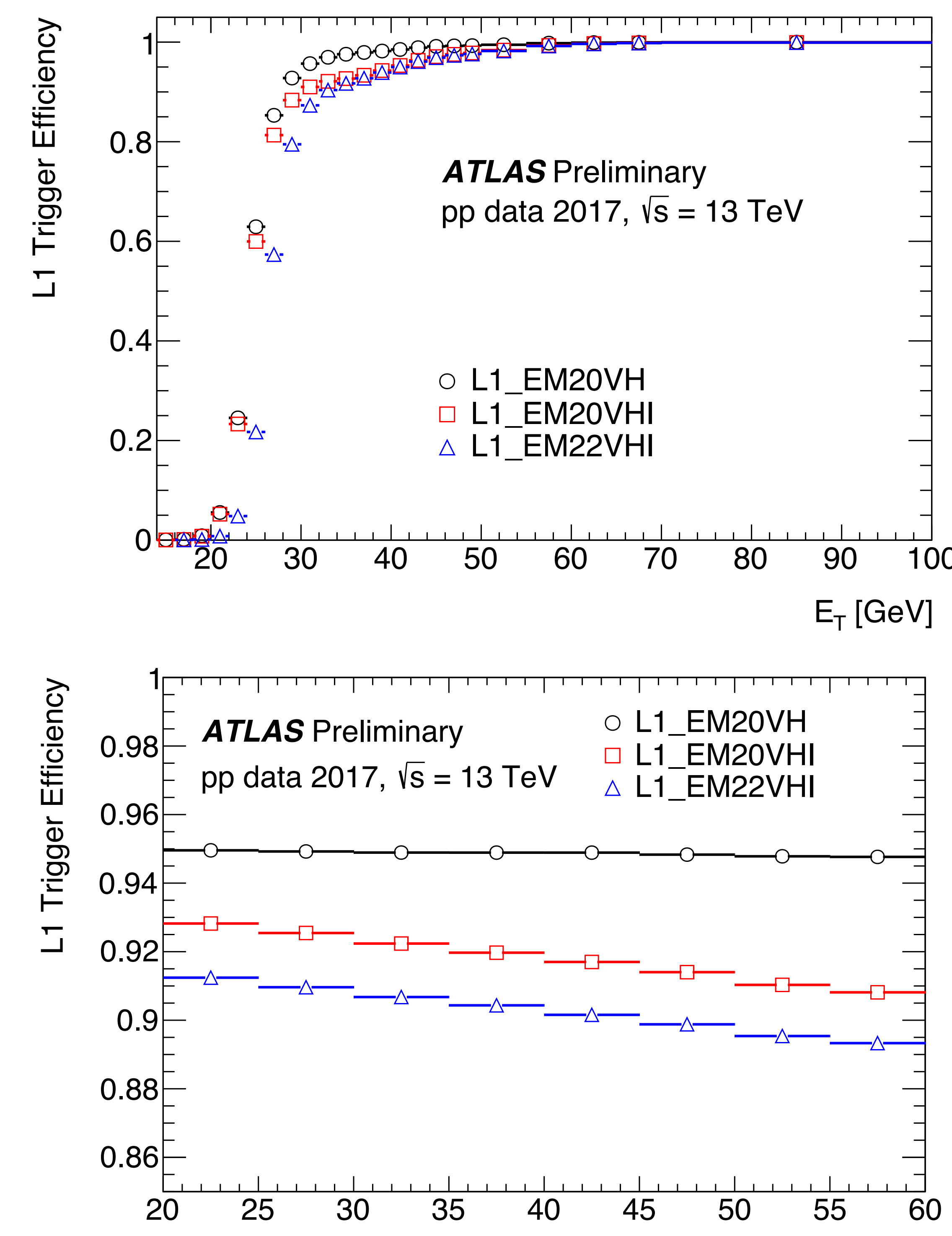


\section{Photon trigger evolution and performance}

\begin{tabular}{|c|c|c|c|c|c|}
\hline & 2015 & 2016 & 2017 & 2018 & \\
\hline $\begin{array}{l}\text { Single } \\
\text { photon }\end{array}$ & g120_loose & \multicolumn{3}{|c|}{ g140_loose } & $\rightarrow$ BSM physics, high ET photons \\
\hline $\begin{array}{l}\text { primary } \\
\text { diphoton }\end{array}$ & \multicolumn{2}{|c|}{ g35_loose_g25_loose } & \multicolumn{2}{|c|}{ g35_medium_825_medium } & $\rightarrow$ Higgs to diphoton \\
\hline $\begin{array}{c}\text { tight } \\
\text { diphoton }\end{array}$ & 2gి20_tight & 2gீ22_tight & \multicolumn{2}{|c|}{ 2g20_tight_icalovloose } & $\rightarrow$ low mass diphoton searches \\
\hline
\end{tabular}
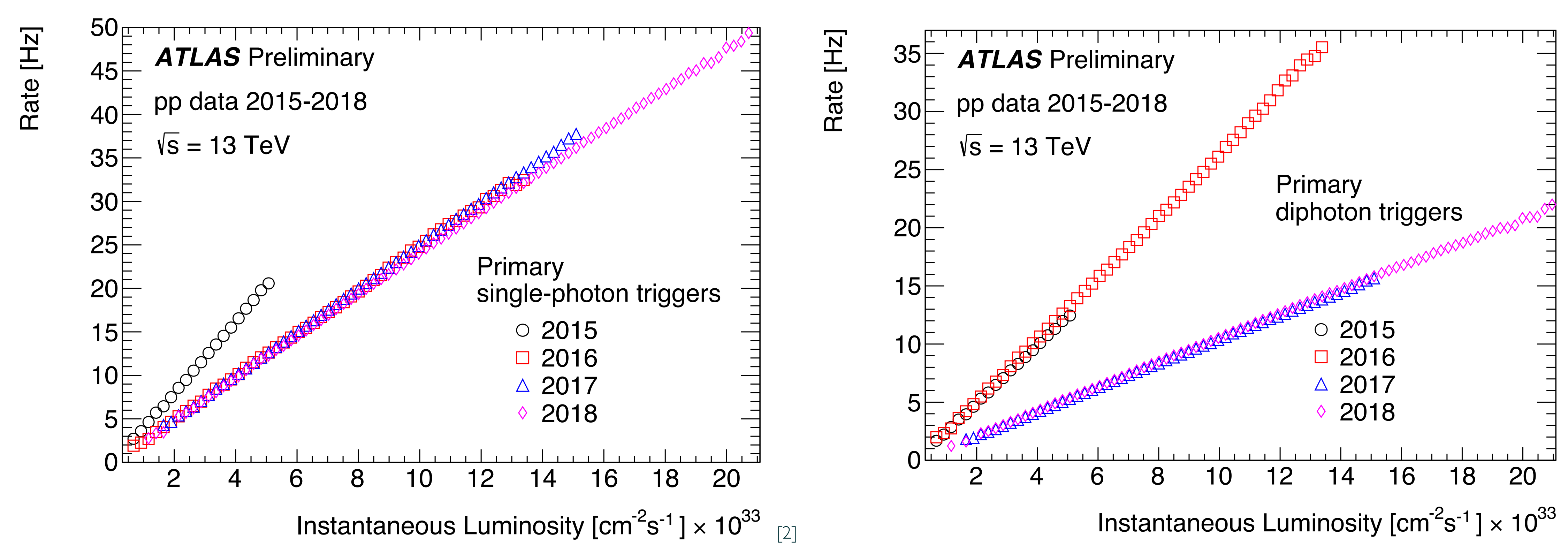


\section{Photon trigger evolution and performance}
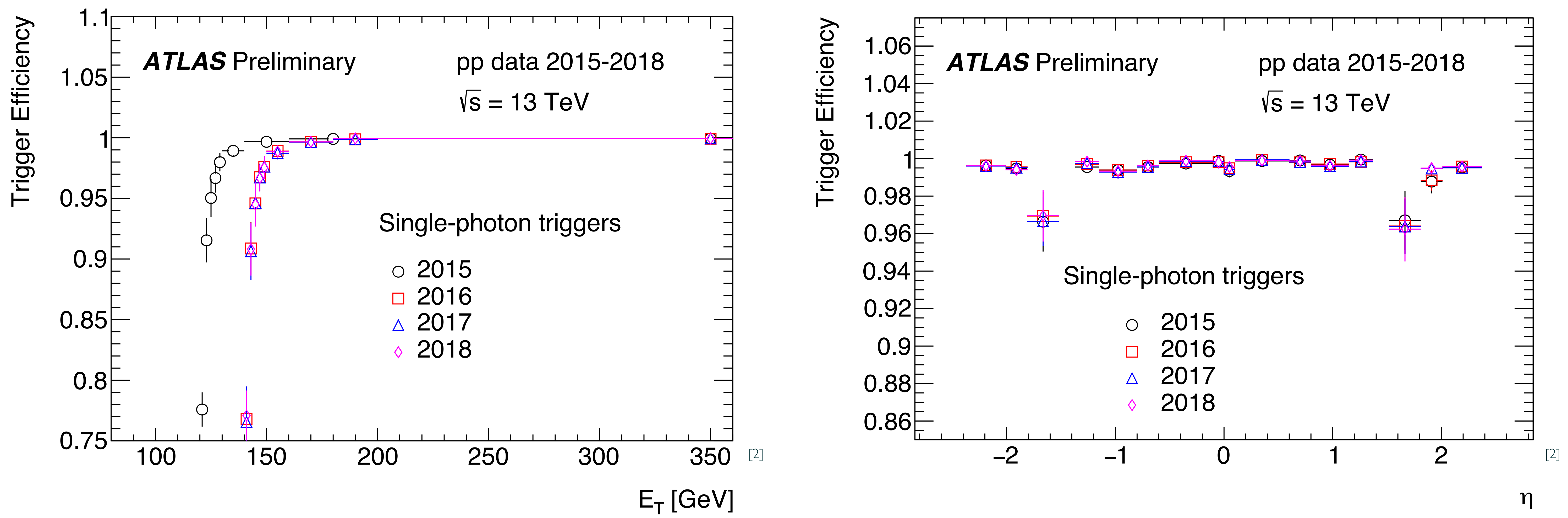

- bootstrap method used to calculate the efficiency

- total uncertainties dominated by systematics, in total $O(1 \%)$

- efficiency in 2016 rises faster due to lower pile-up conditions 


\section{Photon trigger evolution and performance}
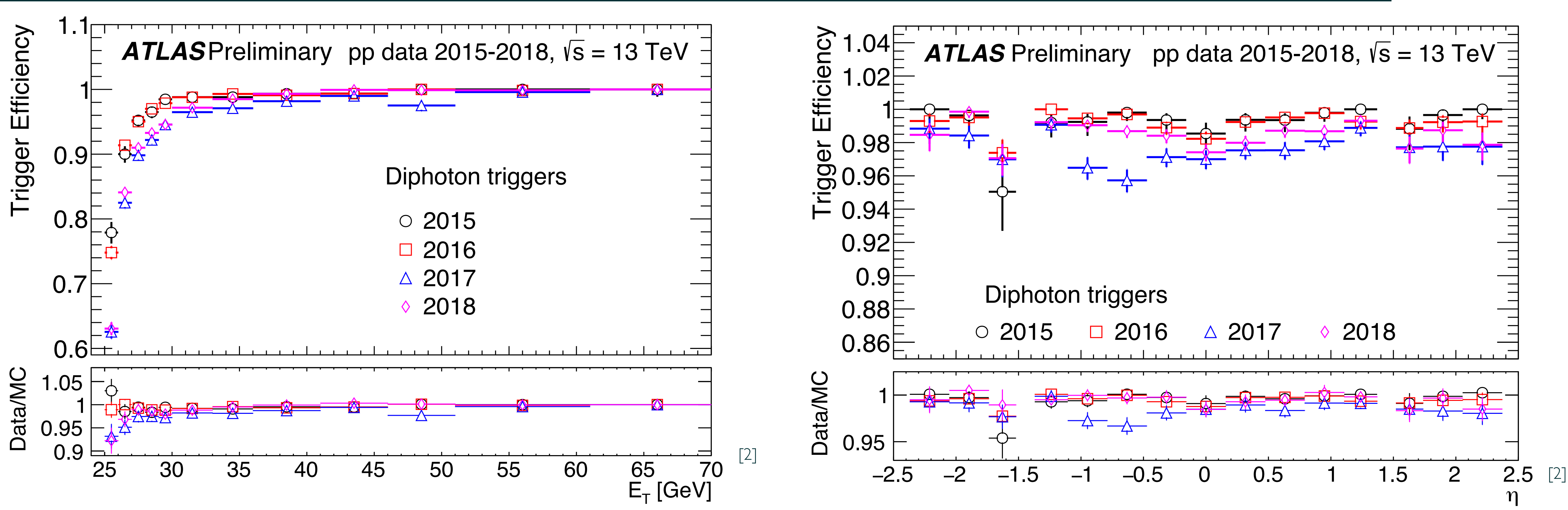

- efficiency measured with radiative $Z$ decay method

- lower efficiency in 2017-2018 due to medium instead of loose online ID

- no pseudorapidity or pile-up dependency in efficiency

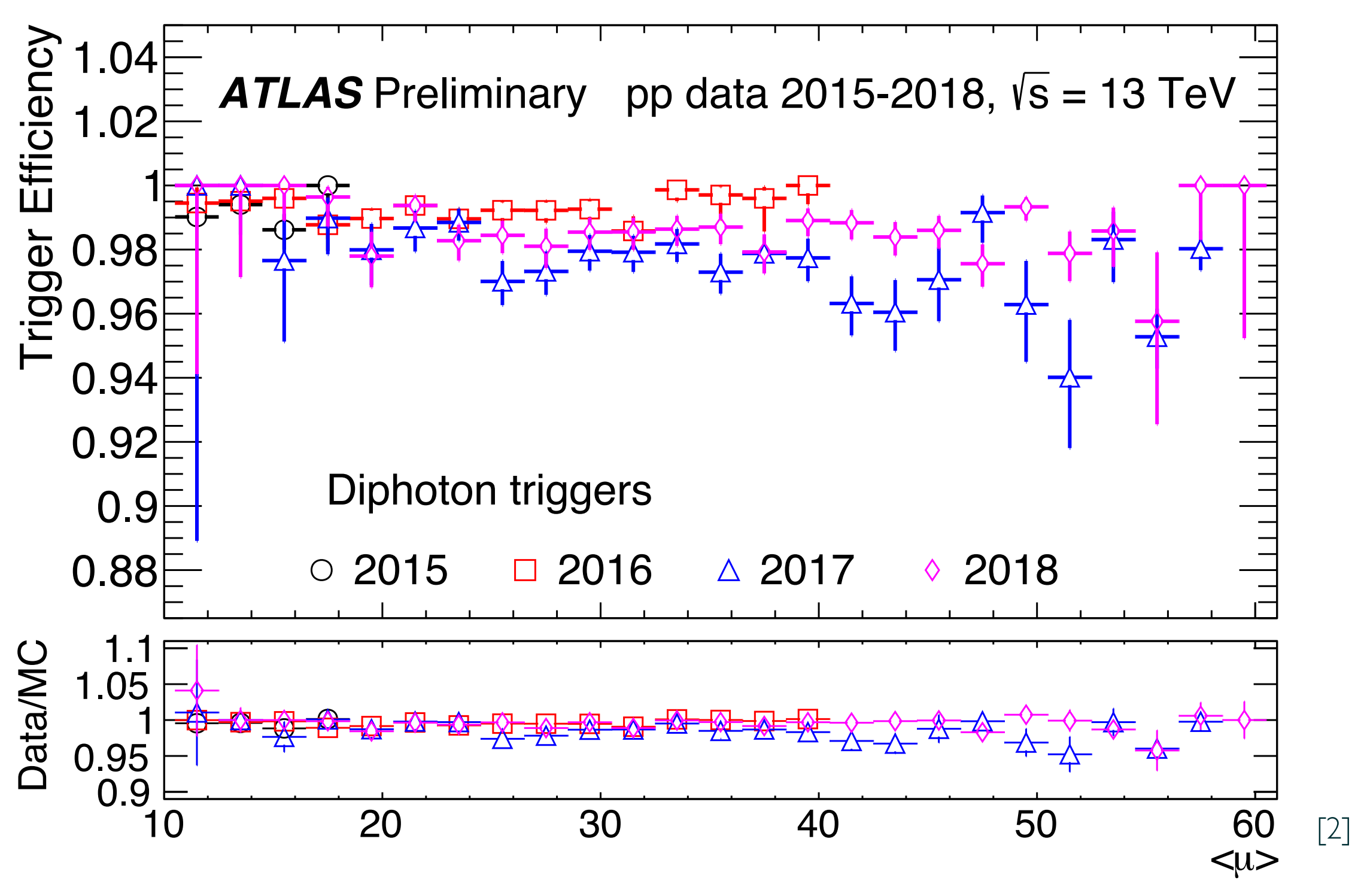




\section{Electron trigger evolution and performance}

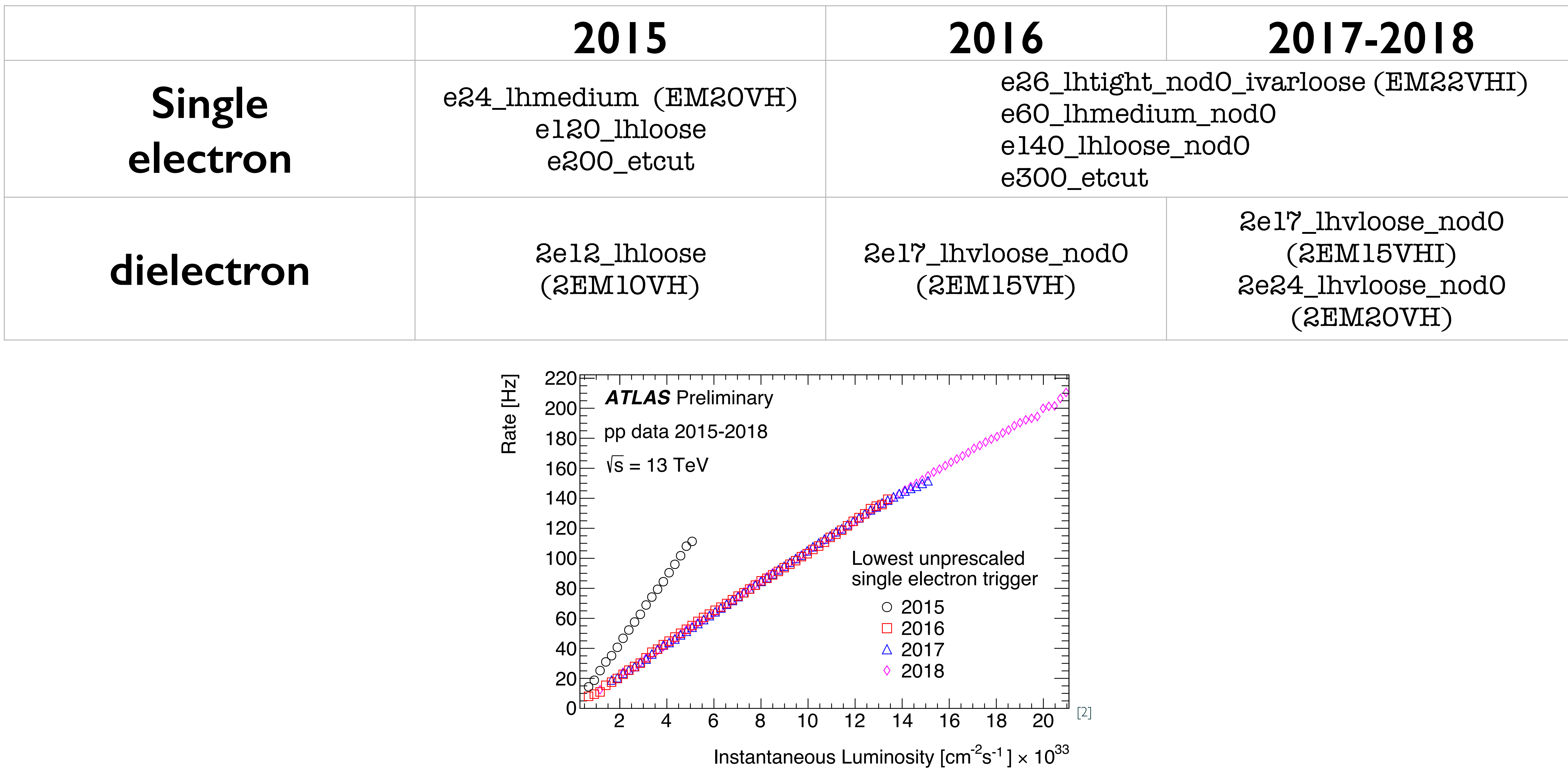




\section{Performance of the Ringer algorithm}

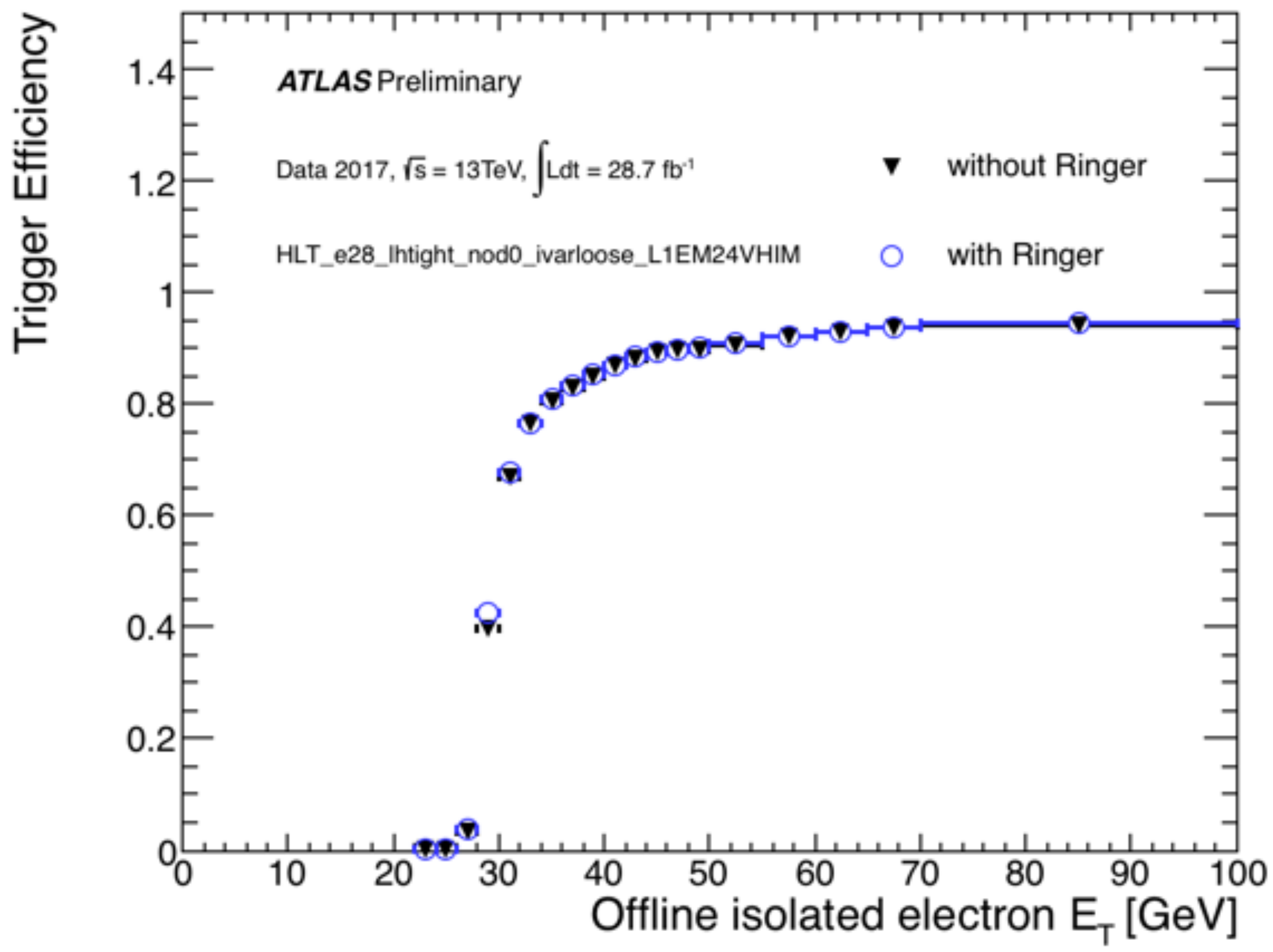

evaluation of Ringer performance in 2017

Comparing Ringer and cutbased selection at fast reconstruction

nearly unchanged efficiency behaviour in single electron case

$50 \%$ CPU reduction for the lowest рт unprescaled single electron trigger 


\section{Performance evolution of single electron trigger}
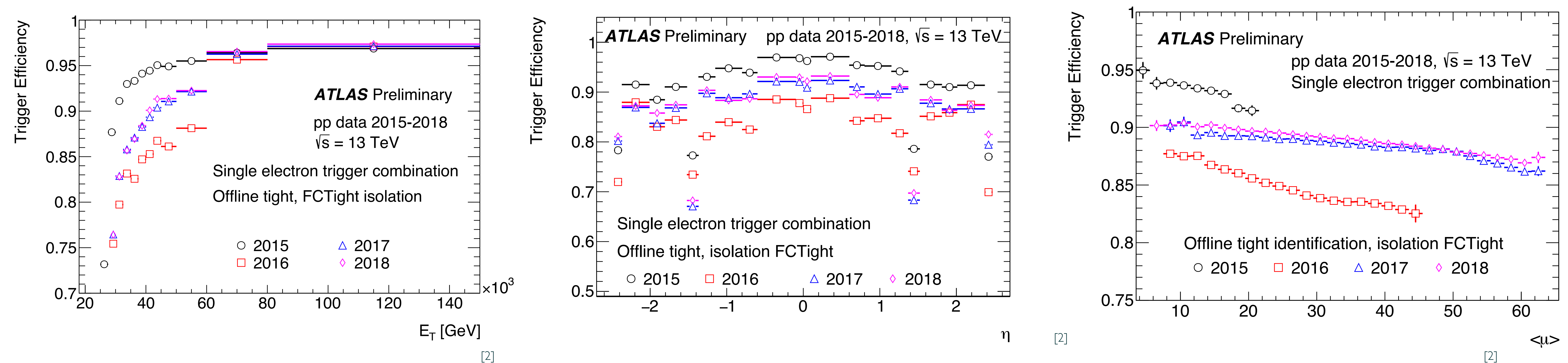

sharper turn on in 2015

lower $\mathrm{E}_{\mathrm{T}}$ threshold, no isolation, looser identification inefficiencies in 2016 below $60 \mathrm{GeV}$ observed

due to $\mathrm{LH}$ calorimeter only selection in precision calorimeter step

2017 data driven likelihood selection, introduction of Ringer algorithm

pile-up dependency reduced towards end of Run-2

residual dependency due to isolation requirements 


\section{photon trigger in heavy ion data taking}

\section{photons}

- photon trigger efficiency evaluated with respect to offline-reconstructed photons

- efficiency shown with and without subtraction of the underlying event

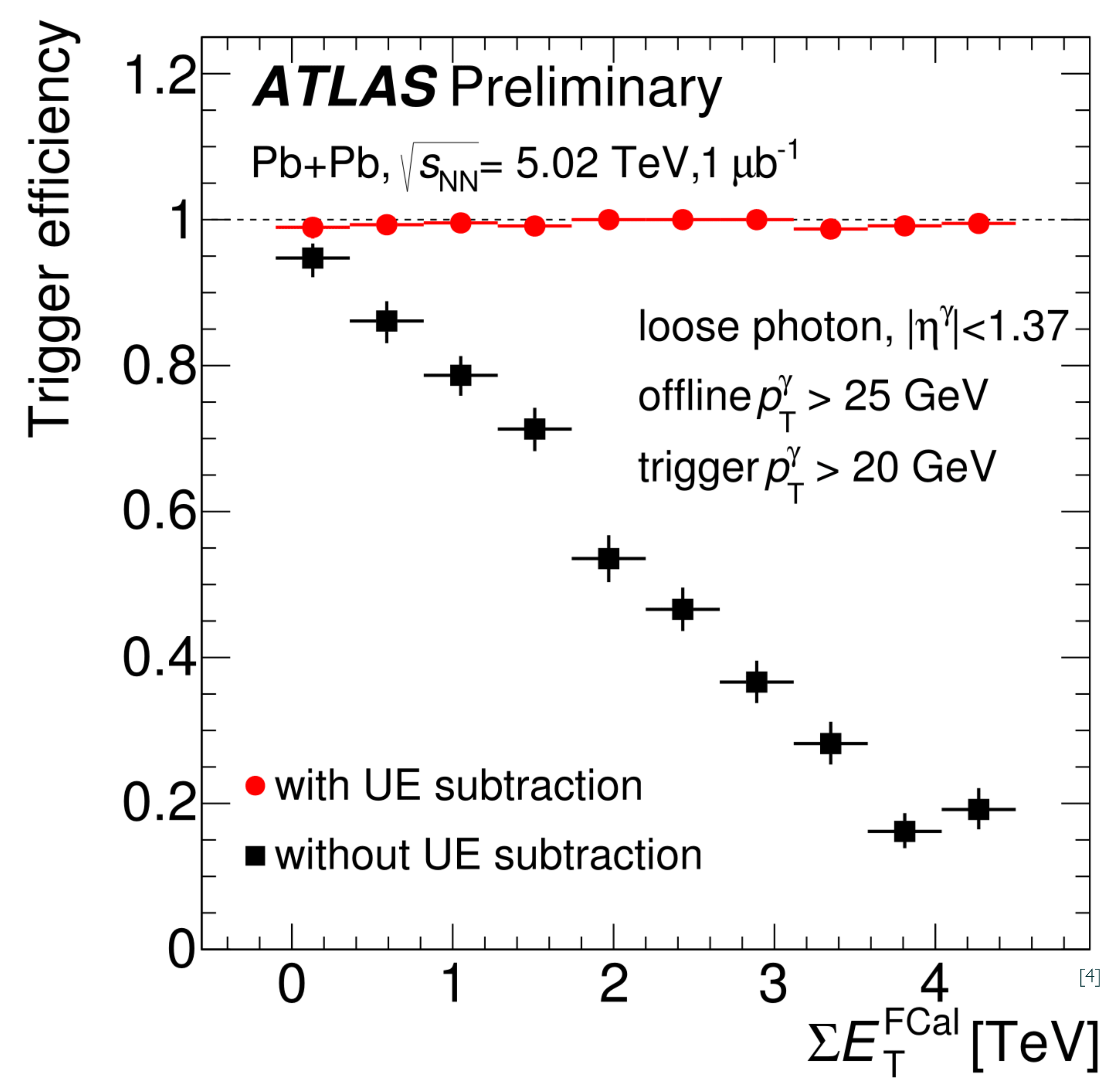




\section{Conclusion and Outlook}

\section{Good understanding of the electron and photon trigger}

performance during Run-2

established methods to measure trigger efficiencies, several improvements during Run-2 to adapt to changing conditions

Many ongoing efforts to improve the electron and photon trigger performance and CPU usage in Run-3

Aim to get closer to offline selections than in Run-2

moving towards multi-threading on trigger level

update of Level-1 Calo electronics with improved granularity, will lead to improved background rejection and better efficiencies 


\section{References}

[I] 'Electron and photon reconstruction and performance in ATLAS using a dynamical, topological cell clustering-based approach' The ATLAS Collaboration, ATL-PHYS-PUB-2017-022

[2] CERN-EP-2019-169

[3] 'Performance of Ringer in Trigger egamma for WCCI20 I8'

https:/twiki.cern.ch/twiki/pub/AtlasPublic/EgammaTriggerPublicResults/HLT_e28_Ihtight_nod0_ivarloose_LI EM24VHIM_et.png

[4] 'Performance of photon triggers for $\mathrm{Pb}+\mathrm{Pb}$ collisions at $5.02 \mathrm{TeV}$ '

https:/twiki.cern.ch/twiki/pub/AtlasPublic/EgammaTriggerPublicResults/PhotonTriggerPerformance_vsFCal_Prelim.png 
BACKUP 


\section{electron reconstruction}

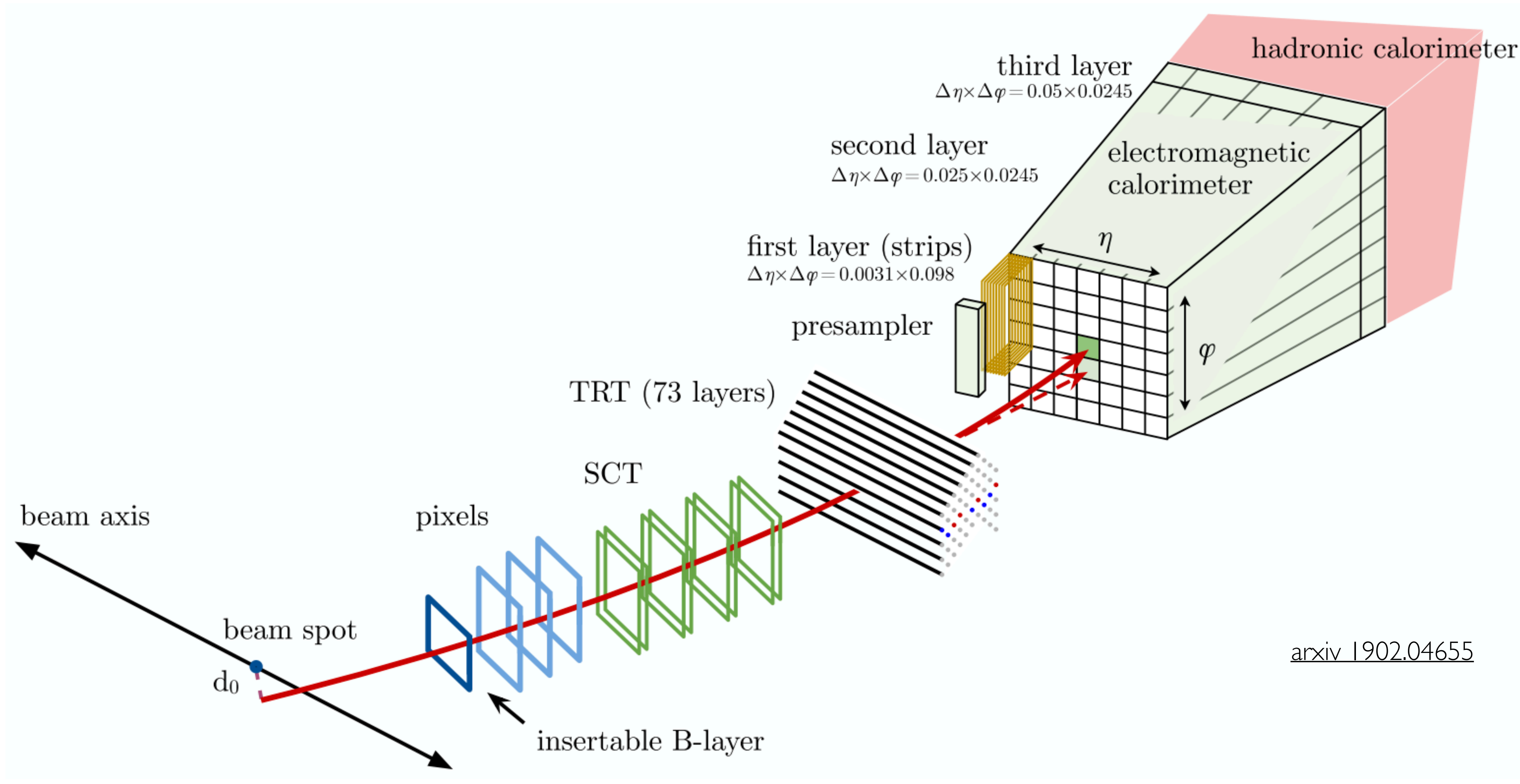




\section{Datasets and simulated samples}

\section{p-p collision data}

$$
\begin{aligned}
& 2015-3.2 \mathrm{fb}^{-1} \\
& 2016-32.9 \mathrm{fb}^{-1} \\
& 2017-43.9 \mathrm{fb}^{-1} \\
& 2018-58.5 \mathrm{fb}^{-1}
\end{aligned}
$$

\section{$\mathbf{P b}$ - Pb collision data}

one month in 2015 and 2018 centre of mass energy per nucleon pair 5.02 TeV

\section{Pb - p collisions}

at 5.02 TeV and 8.16 TeV per nucleon pair in 2016

\begin{tabular}{|c|c|}
\hline process & generators \\
\hline$Z \rightarrow$ ee & $\begin{array}{c}\text { POWHEG-BOX v2 } \\
\text { PYTHIA v8.186 }\end{array}$ \\
\hline$W \rightarrow e v$ & $\begin{array}{c}\text { POWHEG-BOX v2 } \\
\text { PYTHIA v8.186 }\end{array}$ \\
\hline $\mathrm{J} / \psi \rightarrow$ ee & PYTHIA v8.186 \\
\hline $\begin{array}{c}q g \rightarrow q \gamma \\
q \bar{q} \rightarrow q \gamma \\
\text { W/Z production }\end{array}$ & PYTHIA v8.186 \\
\hline$Z \rightarrow \ell \ell \gamma$ & $\begin{array}{l}\text { POWHEG-BOX } \\
\text { PHOTOS } \\
\text { PYTHIA v8.186 }\end{array}$ \\
\hline $\begin{array}{c}\mathrm{qg} \rightarrow \mathrm{q} \gamma \\
\mathrm{q} \overline{\mathrm{q}} \rightarrow \mathrm{gr} \\
\text { QCD dijet }\end{array}$ & PYTHIA v8.186 \\
\hline$H \rightarrow \gamma \gamma$ & PYTHIA v8.186 \\
\hline radion decay & $\begin{array}{c}\text { MADGRAPH5-2.6.0 } \\
\text { PYTHIA v8.212 }\end{array}$ \\
\hline
\end{tabular}

\section{Heavy Ion reference data}

from 5.02 TeV p-p collisions in low pileup conditions 


\section{$Z$ tag and probe measurements}

\section{$Z$ tag and probe method}

tag electron

matched to lowest unprescaled single electron triggers

tight ID, ET $>27 \mathrm{GeV}$

$|\eta|<2.47$, outside the transition region

probe electron

$15 \mathrm{GeV}$ around Z-mass peak opposite sign to tag electron

\section{systematic uncertainties}

- given by varying tag definition, Z-mass window and background subtraction method

- central value is average of variations

- systematic on central value is standard deviation of all variations

- statistical error on central value is average over statistical uncertainty of variations 


\section{trigger inefficiencies}

https:/twiki.cern.ch/twiki/pub/AtlasPublic/EgammaTriggerPublicResults/e26 Ihtight nod0 ivarloose IneffisEMLHTight 20 I7.png

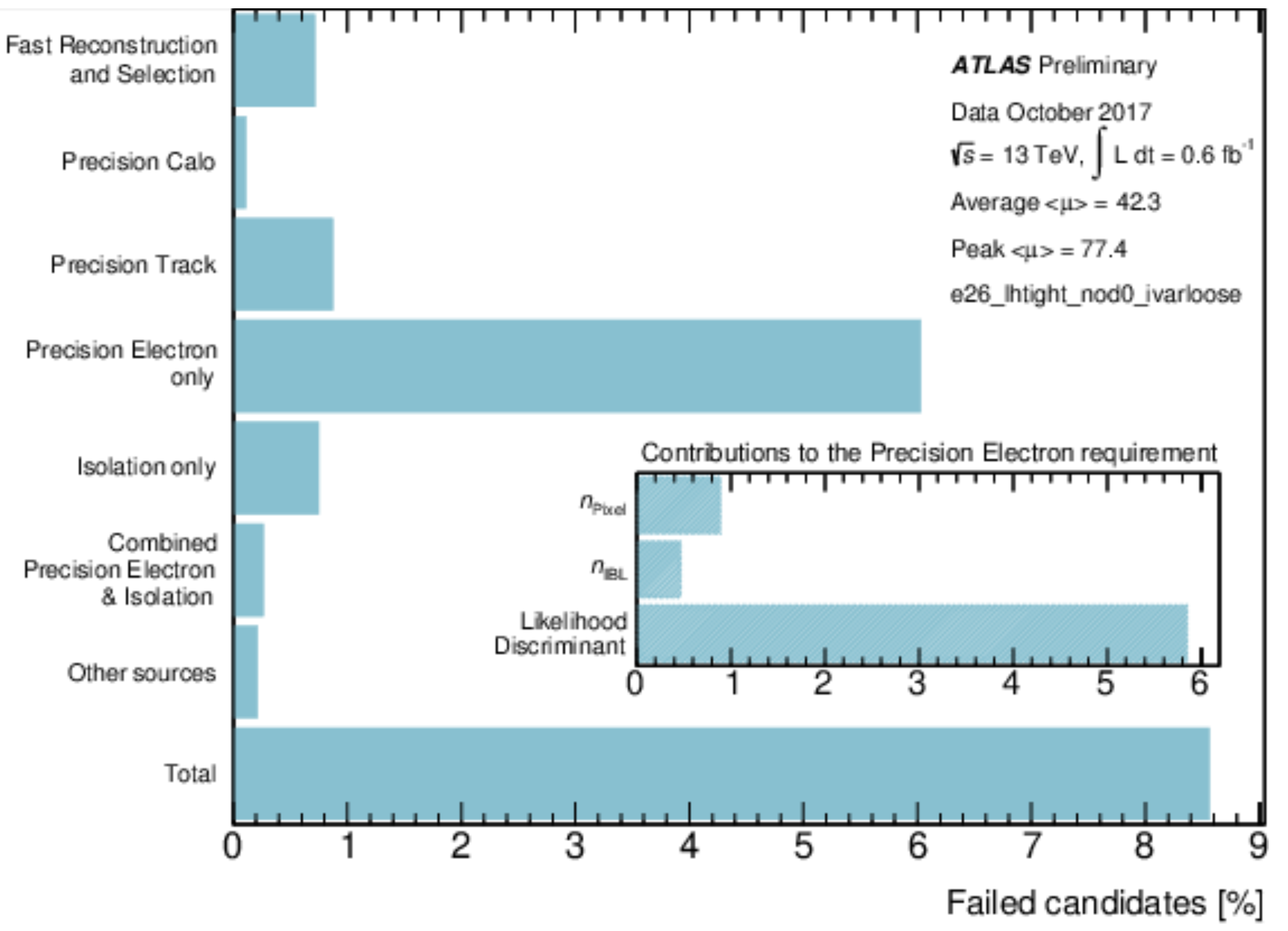

- inefficiency sources for 2017 single electron chain mainly electron identification for tight and isolated trigger
https:/twiki.cern.ch/twiki/pub/AtlasPublic/ EgammaTriggerPublicResults/

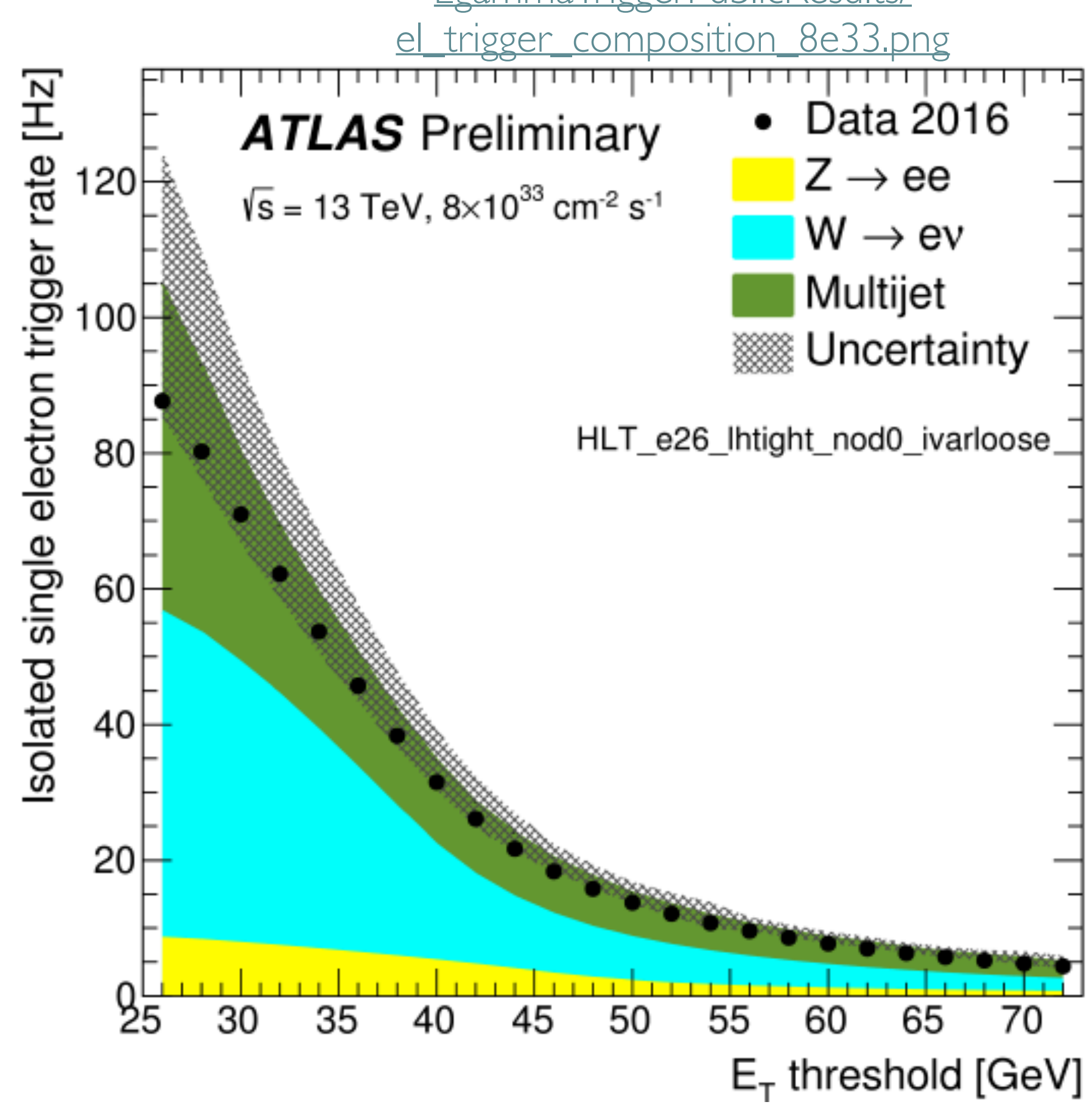

- studies of physics origin of electrons from single electron trigger at HLT shows that main processes are $W$ and $Z$ production 


\section{Trigger reconstruction and identification of photons and electrons}

$2 \times 2$ trigger tower cluster as Rol in EM calorimeter

Level I V:varying $E_{T}$ threshold within -2 and $+3 \mathrm{GeV}$ of nominal threshold

$\mathbf{H}$ : veto on hadronic leakage

I: ET dependent Ecal isolation of cluster

\begin{tabular}{|cl|}
\hline e/ & - precision reconstruction with sliding window algorithm \\
trigger & $\begin{array}{l}\text { - no usage of superclusters } \\
\text { instead of number of primary vertices }\end{array}$ \\
\hline
\end{tabular}

- fast algorithm uses only 2nd layer of EM calo, selection on cluster $\mathrm{E}_{\mathrm{T}}$ and shower shape parameters

Photon - online ID use same cluster shower shapes as the offline ID

trigger (without fudging),

three working points: 'loose', 'medium' (only used online) ,'tight'

- calorimeter only isolation possible

- no tracking information

- fast calorimeter reconstruction cut-based and neural-network ringer algorithm

- precision selection relying on likelihood discriminant, four working

Electron points: Ihvloose, Ihloose, Ihmedium, Ihtight

trigger - additional isolation requirement possible (ivarloose)

- differences w.r.t. offline:

- trigger reconstruction with poorer resolution than offline

- no momentum loss due to bremsstrahlung taken into account
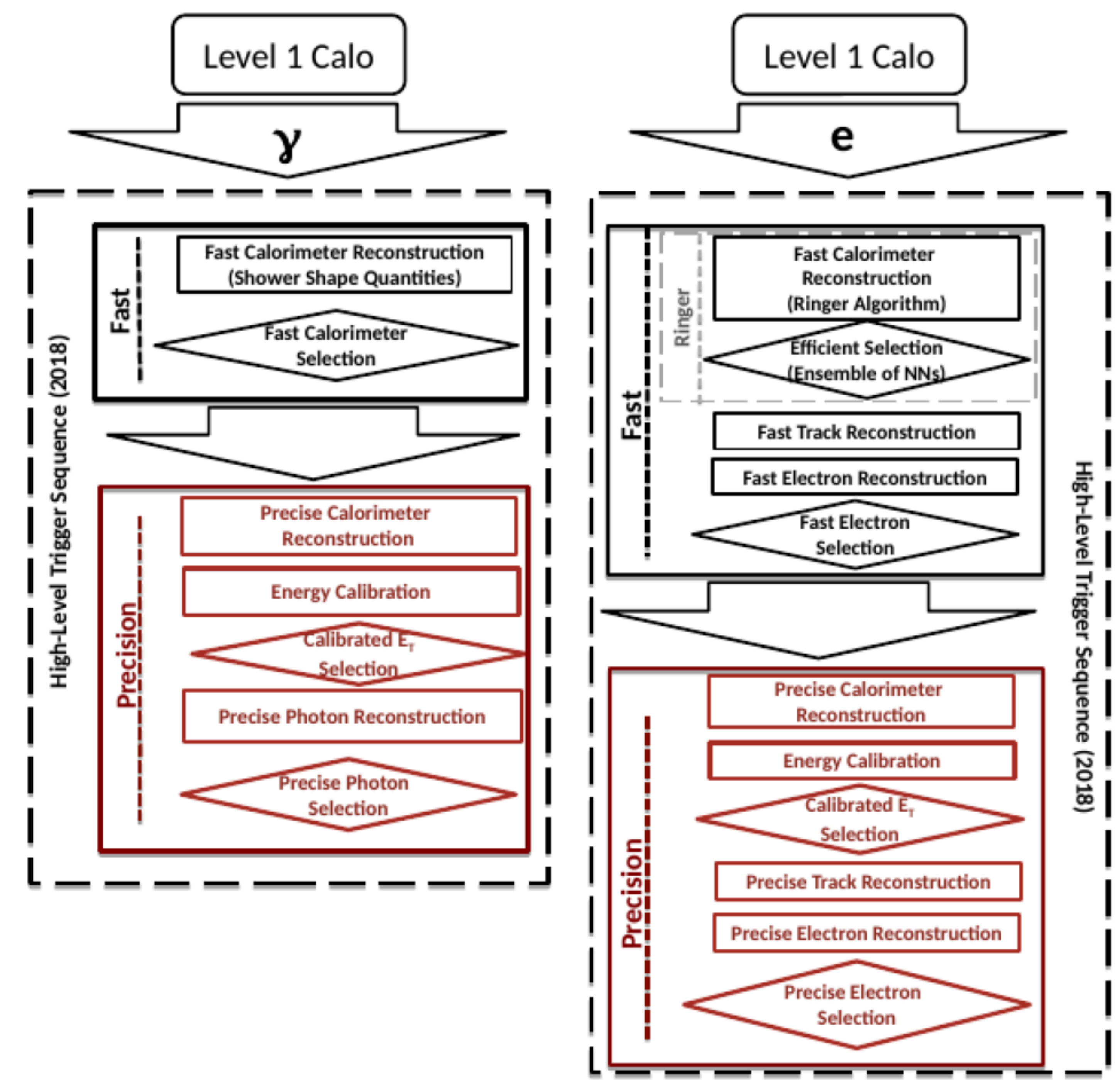


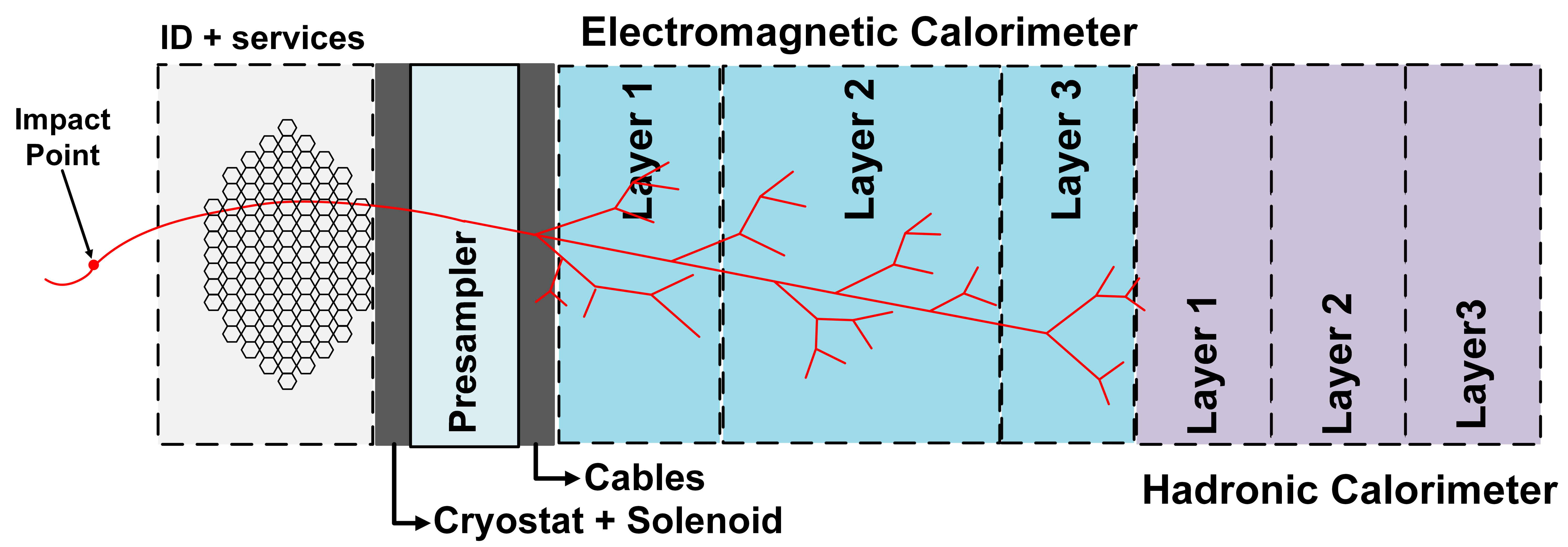

